\title{
Vibrational entropy and microstructural effects on the thermodynamics of partially disordered and ordered $\mathrm{Ni}_{3} \mathrm{~V}$
}

\author{
L. J. Nagel and B. Fultz \\ California Institute of Technology, MS 138-78, Pasadena, California 91125 \\ J. L. Robertson and S. Spooner \\ Oak Ridge National Laboratory, P.O. Box 2008, Oak Ridge, Tennessee 37831 \\ (Received 14 February 1996; revised manuscript received 13 June 1996)
}

\begin{abstract}
Samples of $\mathrm{Ni}_{3} \mathrm{~V}$ were prepared with two microstructures: (1) with equilibrium $D 0_{22}$ order, and (2) with partial disorder (having a large $\mathrm{DO}_{22}$ chemical order parameter, but without the tetragonality of the unit cell). For both materials, we measured the difference in their heat capacities from 60 to $325 \mathrm{~K}$, inelastic neutronscattering spectra at four values of $Q$ at 11 and at $300 \mathrm{~K}$, and Young's moduli and coefficients of thermal expansion. The difference in heat capacity at low temperatures was consistent with a harmonic model using the phonon density of states (DOS) curves determined from the inelastic neutron-scattering spectra. In contrast, at temperatures greater than $160 \mathrm{~K}$ the difference in heat capacity did not approach zero, as expected of harmonic behavior. The temperature dependence of the phonon DOS can be used to approximately account for the anharmonic contributions to the differential heat capacity. We also argue that some of the anharmonic behavior should originate with a microstructural contribution to the heat capacity involving anisotropic thermal contractions of the $D 0_{22}$ structure. We estimate the difference in vibrational entropy between partially disordered and ordered $\mathrm{Ni}_{3} \mathrm{~V}$ to be $S^{\mathrm{pdis}}-S^{\mathrm{ord}}=(+0.038 \pm 0.015) k_{B} /$ atom at $300 \mathrm{~K}$. [S0163-1829(96)02945-1]
\end{abstract}

\section{INTRODUCTION}

There is a growing awareness that vibrational entropy $S_{\text {vibr }}$ can have a significant effect on the thermodynamic stabilities of alloy phases. Vibrational entropy has received sporadic interest over many years. ${ }^{1-6}$ Only recently, however, have studies been performed to measure ${ }^{7-12}$ or calculate accurately ${ }^{13-16}$ the difference in vibrational entropy, $\Delta S_{\mathrm{vib}}^{\beta-\alpha} \equiv S_{\mathrm{vib}}^{\beta}-S_{\mathrm{vib}}^{\alpha}$ between two states, $\alpha$ and $\beta$, of a material. Reported values of $\Delta S_{\mathrm{vib}}^{\beta-\alpha}$ have been in the range of $0.1-0.2 \mathrm{k}_{\mathrm{B}}$ /atom. Such differences in vibrational entropy are large enough to have a considerable influence on critical temperatures, ${ }^{16}$ or the shapes of phase boundaries. ${ }^{11}$

The direct way to determine differences in entropy at constant pressure is to measure differences in heat capacity of two states of a material over a range of temperatures, $\Delta C_{p}(T) \equiv C_{p}^{\beta}-C_{p}^{\alpha}$. This can be done by differential calorimetry, and then calculating:

$$
\Delta S^{\beta-\alpha}(T)=\int_{0}^{T} \frac{\Delta C_{p}\left(T^{\prime}\right)}{T^{\prime}} d T^{\prime}
$$

If the two states of the material remain constant during the measurements, calorimetric methods using Eq. (1) are a good way to obtain $\Delta S_{\mathrm{vib}}^{\beta-\alpha}(T)$. Fortunately, the integrand $\Delta C_{p}(T) / T$ is often largest at low temperatures where there is no significant atomic diffusion, so there are no changes in atomic configurations during the measurements. In the present work we used differential scanning calorimetry to measure $\Delta C_{p}(T)$ for two states of the metallic alloy $\mathrm{Ni}_{3} \mathrm{~V}$. The material was quenched from high temperature to provide a state of partial disorder, which we confirmed to have strong chemical order, but none of the tetragonality of the equilib- rium $D 0_{22}$ structure. ${ }^{17,18}$ The second state of the material was the $D 0_{22}$ equilibrium structure, obtained by annealing the alloy.

The difference in the phonon density of states of the two materials, $g^{\beta}(\varepsilon)-g^{\alpha}(\varepsilon)$, where $\varepsilon$ is the phonon energy, is often the physical origin for differences in $\Delta C_{p}(T)$. The phonon density of states (DOS) of a material can sometimes be measured by inelastic neutron scattering. For transitionmetal aluminides, it was found that the development of optical modes at high energies, associated with vibrations of the Al sublattice in the ordered structure, was the change in the phonon DOS most important to the vibrational entropy. ${ }^{9,10}$ It was also found, however, that interpreting phonon dispersion curves from single crystals of chemically disordered alloys runs the risk of underestimating the density of high-frequency modes. ${ }^{10}$ In the present work we attempted to measure the phonon DOS by a more direct inelastic neutronscattering experiment on polycrystalline samples. We recorded energy-loss spectra at various values of momentum transfer, $Q$, to measure incoherent inelastic scattering and coherent inelastic scattering. The alloy $\mathrm{Ni}_{3} \mathrm{~V}$ proved convenient for this study, since the incoherent scattering cross sections of $\mathrm{Ni}$ and $\mathrm{V}$ are nearly identical, and the lattice dynamics of fcc $\mathrm{Ni}$ are well known. We obtained approximate phonon DOS curves from inelastic neutron-scattering spectra measured at 11 and $300 \mathrm{~K}$. The phonon DOS curves accounted for the low-temperature part of the $\Delta C_{p}(T)$ measured by calorimetry.

The temperature dependence of the phonon DOS curves could account for much of the anharmonic part of $\Delta C_{p}(T)$ measured at temperatures greater than $160 \mathrm{~K}$. Using measured values for thermal expansion and elastic moduli, however, we found a discrepancy in the anharmonic contribution calculated as the classical difference, $C_{p}-C_{V}$. We 
argue that this discrepancy could originate with a microstructural mechanism for storage of elastic energy. The difference in vibrational entropy of the partially disordered and ordered $\mathrm{Ni}_{3} \mathrm{~V}$ was estimated to be $S^{\text {pdis }}-S^{\text {ord }}=(+0.038$ $\pm 0.015) k_{B} /$ atom at $300 \mathrm{~K}$.

\section{PHONON DOS AND HEAT CAPACITY}

We calculate the heat capacity at constant pressure $C_{p}(T)$ as follows. The phonon energy in the lattice $E_{\mathrm{ph}}$ is

$$
E_{\mathrm{ph}}=3 \int_{0}^{\infty} g(\varepsilon) \varepsilon\left(\frac{1}{2}+\frac{1}{e^{\varepsilon / k T}-1}\right) d \varepsilon .
$$

The temperature dependence of $E_{\mathrm{ph}}$ occurs through both the change in the phonon occupancy factor of each mode, and the change in the phonon energies:

$$
\begin{aligned}
\frac{\partial E_{\mathrm{ph}}}{\partial T}= & 3 \int_{0}^{\infty} g_{T_{0}}(\varepsilon) \frac{\varepsilon^{2}}{k T^{2}} \frac{e^{\varepsilon / k T}}{\left(e^{\varepsilon / k T}-1\right)^{2}} d \varepsilon \\
& +3 \int_{0}^{\infty} g_{T_{0}}(\varepsilon) \frac{\partial \varepsilon}{\partial T}\left(\frac{1}{2}+\frac{1}{e^{\varepsilon / k T}-1}\right) d \varepsilon,
\end{aligned}
$$

where $g_{T_{0}}(\varepsilon)$ is the phonon DOS at $T=0$. We recognize the first term in Eq. (3) as the heat capacity at constant volume, $C_{V}(T)$ :

$$
C_{V}(T)=3 \int_{0}^{\infty} g_{T_{0}}(\varepsilon) \frac{\varepsilon^{2}}{k T^{2}} \frac{e^{\varepsilon / k T}}{\left(e^{\varepsilon / k T}-1\right)^{2}} d \varepsilon,
$$

which is equal to $C_{p}(T)$ when the phonon frequencies are unchanged with temperature so that $\partial \varepsilon / \partial T=0$. In what follows, we assume that the individual phonon frequencies undergo small shifts with temperature. We can then obtain the shift in energy of each point on the phonon DOS curve with temperature through $\partial g / \partial T$ and $\partial g / \partial \varepsilon$ :

$$
\frac{\partial E_{\mathrm{ph}}}{\partial T}=C_{V}(T)+3 \int_{0}^{\infty} g_{T_{0}}(\varepsilon) \frac{-\partial g / \partial T}{\partial g / \partial \varepsilon}\left(\frac{1}{2}+\frac{1}{e^{\varepsilon / k T}-1}\right) d \varepsilon .
$$

[When $\partial g / \partial \varepsilon$ is small, it may be important to scale $g(\varepsilon)$ by its breadth.] With increasing temperature there are more phonons in the crystal, increasing the total phonon energy. The phonon energies decrease with the volume of the crystal, however, so with increasing temperature it is advantageous to increase slightly the volume of the crystal, even though this creates an elastic energy, $E_{\mathrm{el}}$ :

$$
E_{\mathrm{el}}=\frac{1}{2} B \triangleleft(3 \alpha T)^{2} .
$$

where $\alpha$ is the linear coefficient of thermal expansion, $B$ is the bulk modulus, and $\sigma$ is the specific volume. This decrease in phonon energy is converted into elastic energy so that

$$
3 \int_{0}^{\infty} g_{T_{0}}(\varepsilon) \frac{-\partial g / \partial T}{\partial g / \partial \varepsilon}\left(\frac{1}{2}+\frac{1}{e^{\varepsilon / k T}-1}\right) d \varepsilon+B a(3 \alpha)^{2} T=0 .
$$

The thermal expansion can be related to the temperature dependence of the phonon DOS through Eq. (7). With the Grüneisen approximation of $\partial \varepsilon / \partial u=-\gamma \varepsilon / u$, where $\gamma$ is a constant:

$$
\begin{gathered}
\frac{\partial g}{\partial T}=-\frac{\partial g}{\partial \varepsilon} \frac{\partial \varepsilon}{\partial \iota} \frac{\partial \iota}{\partial T}, \\
\frac{\partial g}{\partial T}=-\frac{\partial g}{\partial \varepsilon}\left(-\gamma \frac{\varepsilon}{\ddots}\right)(3 \varkappa \alpha) .
\end{gathered}
$$

It is easy to substitute Eq. (9) into Eq. (7) and obtain for the Grüneisen approximation at high temperatures

$$
\alpha=\frac{\gamma C_{V}}{3 B} .
$$

Unfortunately, the Grüneisen approximation is not accurate for the temperature dependence of our experimental $g(\varepsilon)$. Our approach to calculating $C_{V}(T)$ is instead to linearly interpolate the phonon DOS curves of 11 and $300 \mathrm{~K}$, including the zero-point contribution:

$$
\begin{aligned}
C_{V}(T)= & \frac{3 T}{T_{2}-T_{1}} \int_{0}^{\infty}\left[g_{T_{2}}(\varepsilon)-g_{T_{1}}(\varepsilon)\right] \frac{\varepsilon^{2}}{k T^{2}} \frac{e^{\varepsilon / k T}}{\left(e^{\varepsilon / k T}-1\right)^{2}} d \varepsilon \\
& +\frac{3 T}{T_{2}-T_{1}} \int_{0}^{\infty}\left[g_{T_{0}}(\varepsilon)-g_{T_{1}}(\varepsilon)\right] \frac{\varepsilon}{2} d \varepsilon,
\end{aligned}
$$

where the temperatures are $T_{2}=300 \mathrm{~K}$ and $T_{1}=11 \mathrm{~K}$. The phonon DOS at $11 \mathrm{~K}$ is assumed to be the same as that of zero temperature.

To obtain $C_{p}(T)$, we used the result from classical thermodynamics:

$$
C_{p}(T)=C_{V}(T)+9 B \backsim \alpha^{2} T,
$$

where the first term is provided by Eq. (11). To obtain $C_{p}(T)$ from the phonon DOS alone, we obtained the second term from Eq. (7):

$$
\begin{aligned}
C_{p}(T)= & \frac{3 T}{T_{2}-T_{1}} \int_{0}^{\infty}\left[g_{T_{2}}(\varepsilon)-g_{T_{1}}(\varepsilon)\right] \frac{\varepsilon^{2}}{k T^{2}} \frac{e^{\varepsilon / k T}}{\left(e^{\varepsilon / k T}-1\right)^{2}} d \varepsilon \\
& +\frac{3 T}{T_{2}-T_{1}} \int_{0}^{\infty}\left[g_{T_{2}}(\varepsilon)-g_{T_{1}}(\varepsilon)\right] \frac{\varepsilon}{2} d \varepsilon \\
& +3 \int_{0}^{\infty} g_{T_{0}}(\varepsilon) \frac{\partial g / \partial T}{\partial g / \partial \varepsilon}\left(\frac{1}{2}+\frac{1}{e^{\varepsilon / k T}-1}\right) d \varepsilon
\end{aligned}
$$

\section{EXPERIMENTAL}

Ingots of $\mathrm{Ni}_{3} \mathrm{~V}$ were prepared by the induction melting of pieces of elemental $\mathrm{Ni}(99.99+\%)$ and $\mathrm{V}(99.9 \%)$ in an argon atmosphere. The ingots were sealed in evacuated quartz ampoules and homogenized at $1150{ }^{\circ} \mathrm{C}$ for $2 \mathrm{~h}$ and cooled in the furnace. The brittle homogenized ingots were cold-rolled to break them into pieces of about $1 \mathrm{~g}$ mass. These small pieces were annealed in evacuated quartz ampoules at $1150{ }^{\circ} \mathrm{C}$, and quenched by breaking the hot ampoule in iced brine. In what follows, we refer to these materials as "partially disordered." To form the equilibrium $\mathrm{DO}_{22}$ structure, some of these partially disordered pieces were annealed at $850^{\circ} \mathrm{C}$ for $2 \mathrm{~h}$ and cooled in the furnace; we refer to these materials as "ordered." Samples of both materials were heated to $400{ }^{\circ} \mathrm{C}$ and analyzed for evolved hydrogen, oxygen, and nitrogen with a Hewlett-Packard 5890 gas chro- 
matograph equipped with a thermal conductivity detector. None of these gases were detected, and detectability limits were 0.0032 wt $\%$ for hydrogen, 0.078 wt \% for oxygen, and 0.22 wt $\%$ for nitrogen. Chemical compositions and chemical homogeneities were measured with a JEOL Superprobe 733 electron microprobe. Averages of the composition measurements gave 25.44 at $\% \mathrm{~V}$ for the partially disordered alloy, and 25.39 at $\% \mathrm{~V}$ for the ordered alloy. Composition variations within each sample were no more than about 0.1 at $\%$. X-ray diffractometry was performed with an Inel CPS-120 diffractometer using Co $K \alpha$ radiation, and an Al filter was used to suppress $\mathrm{V} K \alpha$ fluorescence.

Low-temperature heat-capacity measurements employed a Perkin-Elmer DSC-4 differential scanning calorimeter (DSC) that had been modified by installing its sample head in a liquid-helium dewar. Masses (about $300 \mathrm{mg}$ ) of the partially disordered and ordered alloys were matched to $0.1 \mathrm{mg}$ accuracy and placed in the two sample pans of the DSC. Heatcapacity measurements comprised pairs of runs, with the two samples interchanged in their sample pans between runs. The difference in heat capacities of the two samples was obtained from the difference of these two sets of runs. To test reproducibility, we obtained nine matched pairs of runs with liquid nitrogen, and two matched pairs with liquid helium as the cryogen. To counteract instrumental drift, runs comprised two pairs of scans over temperature intervals of $30 \mathrm{~K}$, which typically overlapped by $10 \mathrm{~K}$. Scans were performed at 10 and $20 \mathrm{~K} \mathrm{~min}^{-1}$. A measurement was also performed with the ordered sample alone to determine approximately its Debye temperature. Thermal-expansion measurements were performed over a temperature range from 50 to $275^{\circ} \mathrm{C}$ using a Perkin-Elmer TMA7.

Efforts were made to measure ultrasonically the longitudinal and shear wave velocities in the partially disordered and ordered states of $\mathrm{Ni}_{3} \mathrm{~V}$. We used a Panametrics $10 \mathrm{MHz}$ ultrasonic longitudinal wave transducer and a Panametrics 5 $\mathrm{MHz}$ ultrasonic shear wave transducer, whose outputs were passed through a Panametrics Model 5052UA ultrasonic analyzer to a Hewlett Packard 54510A oscilloscope sampling at $1 \mathrm{GHz}$. Two sets of matched cylindrical samples were used. The top and bottom faces of the cylinders were accurately cut parallel and then polished. Successful measurements of the longitudinal and shear wave speeds in $\mathrm{Cu}_{3} \mathrm{Au}$ were made using similar samples from previous calorimetry measurements. ${ }^{12}$ We deduced bulk moduli at room temperature of $18.88 \times 10^{10} \mathrm{~Pa}$ for $L 1_{2}$-ordered $\mathrm{Cu}_{3} \mathrm{Au}$, and $18.70 \times 10^{10} \mathrm{~Pa}$ for chemically disordered fcc $\mathrm{Cu}_{3} \mathrm{Au}$, in good agreement with previous results. ${ }^{19}$ However, none of the $\mathrm{Ni}_{3} \mathrm{~V}$ samples showed a pulse-echo pattern, so measurements were impossible. The samples of $\mathrm{Ni}_{3} \mathrm{~V}$ evidently showed a strong ultrasonic attenuation, perhaps because inhomogeneous internal stresses caused scattering of the ultrasonic waves.

To measure Young's moduli, compression tests were performed using an Instron Model 4204 load frame. A cylindrical sample of $0.55 \mathrm{~cm}$ diameter and $1.9 \mathrm{~cm}$ height was machined from an induction-melted ingot of $\mathrm{Ni}_{3} \mathrm{~V}$, and was heat treated to produce the partially disordered state. After compression testing, the same sample was annealed for $2 \mathrm{~h}$ at $850{ }^{\circ} \mathrm{C}$ and cooled in the furnace to obtain the ordered state, and the compression tests were repeated. The crosshead speed was $0.05 \mathrm{~cm} / \mathrm{min}$, and the extension of the sample was measured using a strain-gauge extensometer. We attempted to perform the compression tests in the elastic regime of the material. The yield stress at room temperature for ordered $\mathrm{Ni}_{3} \mathrm{~V}$ has been reported to be $900 \mathrm{MPa} .{ }^{18}$ Our samples were loaded to only $270 \mathrm{MPa}$, and we ensured that there was no reduction in slope of the stress-strain curves at the highest loads. Several load-unload cycles were performed on each sample. We also performed compression tests on a similar sample of mild steel to confirm that we obtained a reasonable Young's modulus and no hysteresis in our stress-strain measurements.

Samples of the partially disordered and ordered alloys, each about $50 \mathrm{~g}$, were placed in thin-walled aluminum cans and mounted in a closed-cycle helium refrigerator on the goniometer of the HB2 triple-axis spectrometer at the High Flux Isotope Reactor at the Oak Ridge National Laboratory. Measurements on our two materials were performed at both 11 and $300 \mathrm{~K}$. The spectrometer was operated in constant- $Q$ mode with the fixed final energy $E_{f}$ of $14.8 \mathrm{meV}$. The energy-loss spectra were made by scanning the incident energy from 14.8 to $74.8 \mathrm{meV}$. The neutron flux from the monochromator was monitored with a fission detector, which was used to control the counting time for each data point. The incident beam on the pyrolytic graphite monochromator crystal had a collimation of $110^{\prime}$, and $40^{\prime}$ Soller slits were used between the monochromator and the sample. Pyrolytic graphite filters placed after the sample were used to attenuate the $\lambda / 2$ and $\lambda / 3$ contamination. The filtered beam passed through $40^{\prime}$ slits before the pyrolytic graphite analyzer crystal. Following the analyzer, $70^{\prime}$ Soller slits were used before the ${ }^{3} \mathrm{He}$ detector. With this arrangement, the energy resolution varied between 0.9 and $2 \mathrm{meV}$, depending on the energy transfer and the slope of the phonon dispersion surface. Spectra from each specimen were obtained at four values of $Q: \quad 3.48,3.73,3.98$, and $4.23 \AA^{-1}$.

\section{RESULTS}

\section{A. Structure}

Very weak, if any, superlattice diffraction peaks were observed in the Co $K \alpha$ x-ray-diffraction patterns from any samples, but the $\mathrm{x}$-ray form factors are not strongly different for $\mathrm{Ni}$ and $\mathrm{V}$ atoms. On the other hand, neutron diffraction showed strong superlattice diffraction peaks from both the quenched sample and the annealed sample [e.g., the (002), (101), (110) peaks at $\left.q=1.74,1.98,2.51 \AA^{-1}\right]$. From calculated peak intensities, ${ }^{20}$ we found the ordered alloy to have a long-range order (LRO) parameter close to one. The superlattice peaks from the quenched samples were broadened, but had 85 to $90 \%$ of the areas of the superlattice peaks from the annealed sample. The Bragg-Williams LRO parameter of the quenched alloy was therefore between 0.90 and 0.95. X-ray and neutron powder diffractometry showed tetragonality in the annealed specimen that was characteristic of the $D 0_{22}$ structure. Figure 1 shows that the ratio of the intensities of the (200) and (020) diffractions (at $q=3.48$ and $3.55 \AA^{-1}$ ) are $1: 2$, as expected for the powder-averaged intensities of these diffractions. The as-quenched specimens showed no tetragonality, and their sharp diffraction peaks were characteristic of an fcc structure. This is consistent with previous 


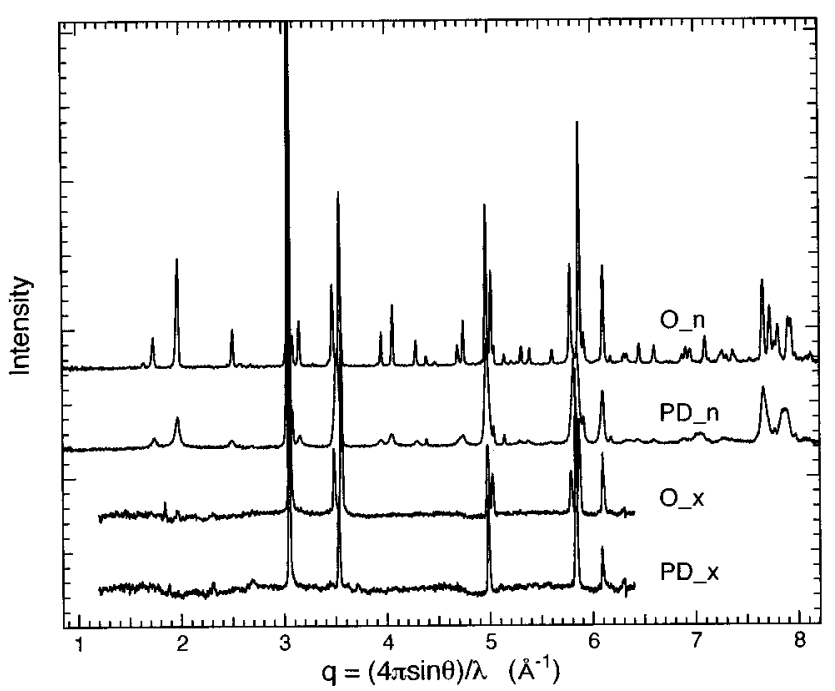

FIG. 1. X-ray (label $\mathrm{x}$ ) and neutron (n) powder-diffraction patterns of the partially disordered (PD) and ordered $(\mathrm{O}) \mathrm{Ni}_{3} \mathrm{~V}$ materials. Some weak diffractions from surface oxides are seen in the $\mathrm{x}$-ray-diffraction patterns, but not in the neutron data.

results of transmission electron microscopy studies that showed a "tweed" microstructure of quenched $\mathrm{Ni}_{3} \mathrm{~V}$. The tweed microstructure has a fine-scale distribution of internal strains, but no regions that are distinctly tetragonal. ${ }^{17,21,22}$ Since the quenched sample had a high degree of the chemical order of the $\mathrm{D}_{22}$ structure, but none of the long-range tetragonality, we call it partially disordered.

\section{B. Calorimetry}

Averaged results from the differential calorimetry measurements are presented in Fig. 2. The sign of the data is positive, showing that $\mathrm{Ni}_{3} \mathrm{~V}$ has a larger heat capacity in the partially disordered state than in the ordered state. The heat capacity was also measured with an ordered sample alone. From this experiment the Debye temperature was determined to be approximately $365 \mathrm{~K}$. [This could be inaccurate, since data were not obtained much below $100 \mathrm{~K}$, but it showed reasonable agreement with the heat capacity calculated with the phonon DOS curves as described below, and should be adequate for our need to account for the low-temperature part of $\Delta C_{p}(T)$ for use in Eq. (1).] Using this Debye temperature of $365 \mathrm{~K}$ for the ordered sample, the data of Fig. 2 could be best fit at temperatures below $160 \mathrm{~K}$ with a Debye temperature of $360 \mathrm{~K}$ for the partially disordered sample. At high temperatures, these Debye temperatures provide a larger vibrational entropy for the partially disordered than the ordered alloy, and $\Delta S_{\text {vib }}$ is

$$
\Delta S_{\text {vib }}=3 k_{B} \ln \left(\frac{365}{360}\right)=0.041 \quad k_{B} / \text { atom. }
$$

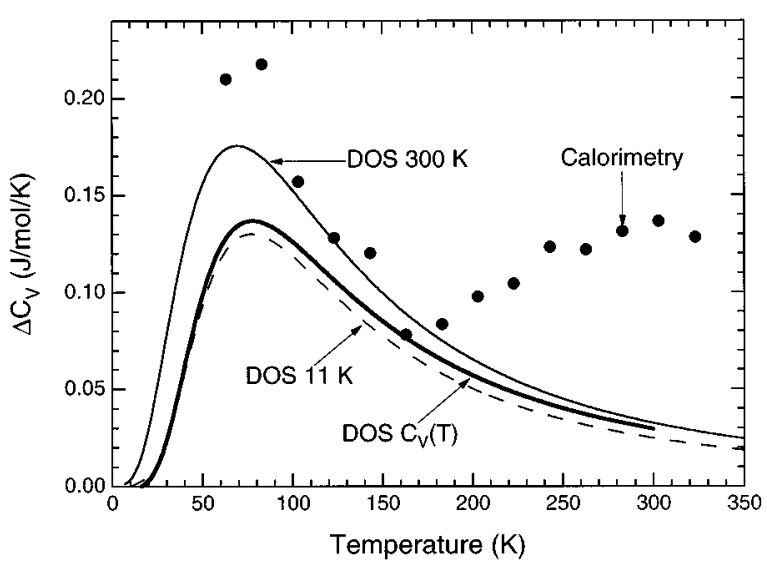

FIG. 2. Differential heat capacities. Points: averages of scans with differential calorimetry of the difference in heat capacity of partially disordered and ordered $\mathrm{Ni}_{3} \mathrm{~V}$ materials (sign is partially disordered minus ordered). Variations between different runs were $\pm 0.025 \mathrm{~J} / \mathrm{mol} / \mathrm{K}$. Lines: calculations as described in the text.

\section{Elastic moduli, thermal-expansion coefficients, Grüneisen constants}

The stress-strain curves of Fig. 3 show considerable hysteresis. For determining the Young's modulus we used the unloading curve at stresses slightly below the peak stress of the test. We expect this slope to be least affected by internal processes contributing to hysteresis, and more representative of the elastic response. In support of this expectation, we found that the unloading curve was more reproducible than the loading curve, and we found that the Young's modulus of the ordered material was in good agreement with that of François, Hug, and Veyssière. ${ }^{18}$ Young's moduli are presented in Table I, together with linear coefficients of thermal expansion.

Grüneisen constants were obtained from the temperature dependence of the phonon DOS. For the ordered alloy, for example, we rescaled in energy the $300 \mathrm{~K}$ phonon DOS curve by $0.95 \%$ to achieve a best match with the $11 \mathrm{~K}$ curve. The $300 \mathrm{~K}$ curve for the partially disordered alloy matched best with the $11 \mathrm{~K}$ curve for a rescaling by $1.5 \%$. Using the linear coefficients of thermal expansion in Table I with the $289 \mathrm{~K}$ difference between 11 and $300 \mathrm{~K}$, we obtain the Grüneisen constants $\gamma$ of 4.9 for the partially disordered alloy and 3.2 for the ordered alloy. These values seem large, perhaps because the measured thermal contraction is an average of anisotropic constants involving different crystallographic directions. On the other hand, using Eq. (10) we obtain Grüneisen constants of 1.15 for the partially disordered material and 1.53 for the ordered material. Not only are these Grüneisen constants smaller that those obtained from the phonon DOS, but their relative sizes are reversed for the two mate-

TABLE I. Measured physical properties of partially disordered and ordered $\mathrm{Ni}_{3} \mathrm{~V}$.

\begin{tabular}{lcccc}
\hline \hline State of $\mathrm{Ni}_{3} \mathrm{~V}$ & Young's modulus & $\alpha(350 \mathrm{~K})$ & $\gamma$ [from Eq. (10)] & $\gamma$ (from DOS) \\
\hline Partially disordered & $12.4 \times 10^{10} \mathrm{~Pa}$ & $10.6 \times 10^{-6} \mathrm{~K}^{-1}$ & 1.15 & 4.9 \\
$\mathrm{D}_{22}$ ordered & $17.2 \times 10^{10} \mathrm{~Pa}$ & $10.2 \times 10^{-6} \mathrm{~K}^{-1}$ & 1.53 & 3.2 \\
\hline \hline
\end{tabular}




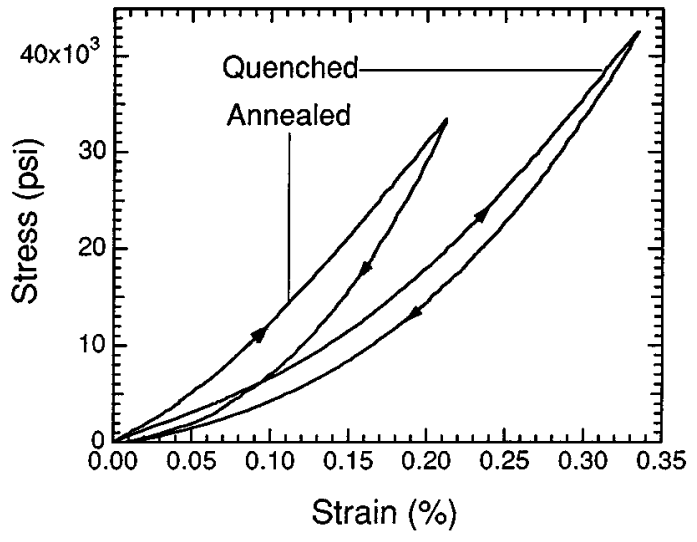

FIG. 3. Engineering stress-strain curves from compression tests of partially disordered and ordered $\mathrm{Ni}_{3} \mathrm{~V}$. These curves were the fifth load-unload cycles of the materials.

rials. We believe that the inconsistency in these Grüneisen constants is caused by the crystallographic anisotropy of $\mathrm{Ni}_{3} \mathrm{~V}$.

\section{Inelastic neutron scattering}

The 16 inelastic-scattering spectra from the two samples (partially disordered and ordered), at two temperatures (11 and $300 \mathrm{~K})$, and four values of $Q(3.48,3.73,3.98,4.23$ $\AA^{-1}$ ), are presented in Fig. 4. The strong elastic peak is seen at energies below $2 \mathrm{meV}$. At higher energy losses, the inelastic scattering shows features of the phonon DOS of the material, such as the peak from the longitudinal branch at 33 $\mathrm{meV}$. In each of these spectra, the phonon DOS is significantly modified by a thermal factor and the $Q$ dependence of the coherent inelastic scattering. Since all spectra were obtained with the same total incident beam flux, the thermal factor is evident from the stronger scattering at 300 than at $11 \mathrm{~K}$. The $Q$ dependence of the coherent inelastic scattering is seen as differences in the shapes of the four spectra in each of the four graphs of Fig. 4. Overlays of these raw data show that the inelastic loss spectra of the ordered alloy are at slightly higher energies than are spectra of the disordered alloy, and the inelastic loss spectra of both materials show some thermal softening.

We proceeded to analyze these data to extract four phonon DOS curves for the two samples, partially disordered and ordered, at two temperatures, 11 and $300 \mathrm{~K}$. Our method has similarities to approaches used previously. ${ }^{10,23-25}$ To understand the effect of temperature, we calculated the incoherent inelastic scattering using the conventional multiphonon expansion. ${ }^{26-28}$ The calculation was performed for $300 \mathrm{~K}$ with the phonon DOS curve of fcc Ni. ${ }^{10,29,30}$ The results showed that at the relatively low values of $Q$ and temperature of the present experiments, the inelastic scattering is strongly dominated by one-phonon processes, but the small background enhancement of the $300 \mathrm{~K}$ data with respect to the $11 \mathrm{~K}$ data was approximately consistent with the calculated two phonon scattering. First, a background was stripped from each spectrum. Second, each spectrum was corrected by the one-phonon thermal factor, $f(T)$, of

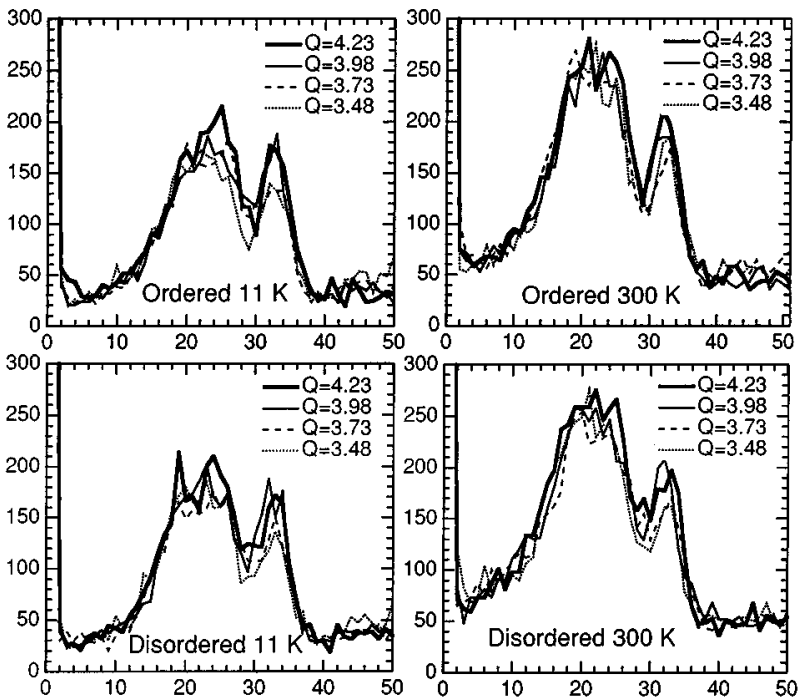

FIG. 4. Raw neutron energy-loss spectra for the partially disordered and ordered $\mathrm{Ni}_{3} \mathrm{~V}$ powders, obtained at four values of $Q$ and two temperatures as indicated.

$$
f(T)=\varepsilon[1-\exp (-\varepsilon / k T)] .
$$

All spectra were then normalized to unit area. Finally, for each specimen at each temperature, we summed all four spectra for the different values of $Q$. For the incoherent inelastic part of the scattering, this data processing procedure should provide the total phonon DOS, since the incoherent scattering from both $\mathrm{Ni}$ and $\mathrm{V}$ atoms are the same. The coherent inelastic scattering from $\mathrm{Ni}$ atoms contributes significantly to the spectra, however.

It is usually not possible to obtain an accurate phonon DOS from coherent inelastic scattering spectra of polycrystals without prior knowledge of the lattice dynamics of the material, ${ }^{31}$ and there is little such knowledge for $\mathrm{Ni}_{3} \mathrm{~V}$. One problem occurs because $\mathrm{Ni}$ and $\mathrm{V}$ atoms have different coherent scattering cross sections. In particular, we were concerned initially that the negligible coherent scattering from $\mathrm{V}$ atoms will deemphasize vibrational modes having polarization eigenvectors with large components for $\mathrm{V}$ atoms. Highfrequency optical modes should be particularly susceptible to this problem. However, if there were significant differences between samples in vibrational modes emphasizing $\mathrm{V}$ atoms, we should be able to detect the differences through changes in the incoherent inelastic scattering. Furthermore, most optical modes involve some motion of Ni atoms that are neighbors of the $\mathrm{V}$ atoms, so these modes should also contribute to the coherent inelastic scattering. We examined carefully the spectra in Fig. 4 and the final DOS curves of Fig. 5 over the energy range of $25-50 \mathrm{meV}$. We found no distinct differences in the features of curves for the four sets of data.

The processed phonon DOS curves are very similar to each other, and to the phonon DOS curve for fcc $\mathrm{Ni}^{10}{ }^{10}$ In our previous work, ${ }^{10}$ a Born-von Kármán model was used with interatomic force constants of fcc Ni to calculate the eigenfrequencies and eigenvectors of the dynamical matrix. With this information for each phonon in the first Brillouin zone, the coherent inelastic scattering was calculated by performing a crystallographic average of the dynamical structure fac- 


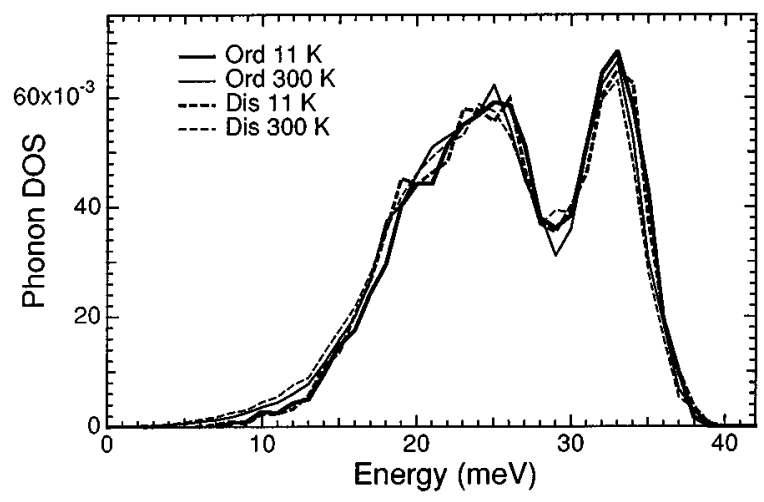

FIG. 5. Phonon DOS obtained from the experimental data of Fig. 2 by processing as described in the text.

tor intensity at explicit values of $\mathbf{Q}$ with respect to the crystallographic axes. It was shown that for fcc $\mathrm{Ni}$, summing the dynamical structure factors for the four values of $Q(3.48$, $3.73,3.98,4.23 \AA^{-1}$ ) provides a good approximation to the phonon DOS. ${ }^{10}$ More significantly, systematic errors in the analysis are not too important for our purposes, since our need is to identify differences in the phonon DOS curves for the different states of the material.

Owing to the excellent $0.01 \mathrm{meV}$ energy accuracy and stability of the HB2 spectrometer, small energy shifts in the phonon DOS curves can be measured reliably. It is seen in Fig. 5 that the curve for the ordered alloy at $11 \mathrm{~K}$ is shifted to the highest energy, and the lowest curve is for the disordered alloy at $300 \mathrm{~K}$. Differences in the partially disordered and ordered DOS curves at the same temperature are indicative of the harmonic contribution to the difference in vibrational entropy between the two materials, and the changes of these differences with temperature are indicative of anharmonic effects on the difference in vibrational entropy.

\section{DISCUSSION}

\section{A. Anharmonicity and phonon DOS}

With Eq. (4), we calculated the harmonic differential heat capacity, $\Delta C_{V}(T) \equiv C_{V}^{\mathrm{PD}}(T)-C_{V}^{(\mathrm{O})}(T)$, from the differences in phonon DOS of the partially disordered (PD) and ordered (O) samples. The calculation of $\Delta C_{V}(T)$ with the $11 \mathrm{~K}$ phonon DOS curves is presented as a thin dashed curve in Fig. 2, and the calculation of $\Delta C_{V}(T)$ from the $300 \mathrm{~K}$ phonon DOS curves is presented as a thin solid curve. The measured differential heat capacity, $\Delta C_{p}(T)$, at temperatures below 160 $\mathrm{K}$ is described adequately by calculations with a harmonic model using either pair of phonon DOS curves (11 or 300 $\mathrm{K})$. At temperatures greater than $160 \mathrm{~K}$, however, the calculated curves are qualitatively incorrect. The $\Delta C_{p}(T)$ measured by calorimetry did not asymptotically approach zero, as expected in a harmonic model where the heat capacities of both states of the material approach the same Dulong-Petit limit of $3 \mathrm{k}_{\mathrm{B}}$ /atom.

With Eq. (11) we calculated $C_{V}(T)$ for both the partially disordered and ordered alloys, using the phonon DOS curves shown in Fig. 5. The result for $\Delta C_{V}(T)$ is presented as a thick curve in Fig. 2. At low temperatures, this new $\Delta C_{V}(T)$ curve is much like the $\Delta C_{V}(T)$ curve from the $11 \mathrm{~K}$ phonon DOS, and near $300 \mathrm{~K}$ the new $\Delta C_{V}(T)$ curve approaches the $\Delta C_{V}(T)$ curve from the $300 \mathrm{~K}$ phonon DOS. At $300 \mathrm{~K}$, the new $\Delta C_{V}(T)$ curve lies below the harmonic $\Delta C_{V}(T)$ curve from the $300 \mathrm{~K}$ phonon DOS, however, owing to the reduction of zero-point energy accompanying the softening of the phonon DOS.

Figure 2 shows that for temperatures greater than $160 \mathrm{~K}$, there is poor agreement between the $\Delta C_{V}(T)$ curve calculated with Eq. (11) and the data measured by calorimetry. Since the energy scale for the HB2 spectrometer is reliable, the heat capacities calculated with Eq. (11) should be accurate, since they are integral quantities over the phonon DOS. Modifications of Eq. (11) that started the transition from the low-temperature DOS to the high-temperature DOS at 160 $\mathrm{K}$, for example, were also incapable of providing the large anharmonic behavior in $\Delta C_{p}(T)$ measured by calorimetry.

Our first attempt to include the anharmonic effects of thermal expansion on the heat capacity used Eq. (12) with the values of $\alpha$ from Table I, and $v=6.8 \times 10^{-6} \mathrm{~m}^{3} / \mathrm{mol}$ from the diffraction data. We assume that the Young's modulus of Table I is the same as the bulk modulus, $B$. This is valid for isotropic materials having a Poisson's ratio of $1 / 3$, and the Poisson's ratio of microtwinned $\mathrm{Ni}_{3} \mathrm{~V}$ is reported to be $0.354{ }^{18}$ The difference in anharmonic heat capacity, $\Delta C_{\mathrm{anh}}(T)=C_{\mathrm{anh}}^{\mathrm{PD}}(T)-C_{\mathrm{anh}}^{\mathrm{O}}(T) \quad$ was calculated to be $\Delta C_{\text {anh }}(T)=-2.43 \times 10^{-4} T(\mathrm{~J} / \mathrm{mol} / \mathrm{K})$. This expression predicts the wrong sign for the $\Delta C_{\mathrm{anh}}(T)$ (primarily because the elastic modulus of the partially disordered sample is smaller than that of the ordered sample). At the temperature of 300 $\mathrm{K}$, adding $\Delta C_{\text {anh }}(300)=-0.073 \mathrm{~J} / \mathrm{mol} / \mathrm{K}$ to the $\Delta C_{V}(T)$ curves in Fig. 2 would provide a total differential heat capacity with a negative sign in Fig. 6.

Our second attempt to include anharmonic effects of thermal expansion on the heat capacity was to use only the phonon DOS curves through Eq. (13). The third term of Eq. (13) was calculated with the phonon DOS curves for both the partially disordered and ordered alloys, and their difference was taken. There was a problem of how to define $\partial g / \partial T$ with the DOS curves obtained at 11 and $300 \mathrm{~K}$, since the thermal expansion and $\partial g / \partial T$ will approach zero at low temperatures. We assumed that $\partial g / \partial T$ would track the harmonic heat capacity of the alloy between 11 and $300 \mathrm{~K}$, so at $300 \mathrm{~K}$ we defined $\partial g / \partial T$ as the difference in the DOS curves measured at 300 and $11 \mathrm{~K}$, divided by $192 \mathrm{~K}$. For lower temperatures, we reduced $\partial g / \partial T$ in proportion to the ratio of the heat capacity at temperature to the heat capacity at $300 \mathrm{~K}$. The biggest problem with the use of Eq. (13), however, was that there were some energies where $\partial g / \partial E$ was nearly zero, causing large values of the integrand in the third term of Eq. (13). It was necessary to suppress those points where $\partial g / \partial E$ was nearly zero. Variations in this practice caused uncertainties in the anharmonic part of the differential heat capacity of about $\pm 20 \%$. This differential anharmonic contribution is presented in Fig. 6, labeled as "DOS Anharmonic." Adding this anharmonic contribution to the $\Delta C_{V}$ from Eq. (12) provides the total differential heat capacity labeled "DOS $\Delta C_{p}{ }^{\prime \prime}$ in Fig. 6. The calculated $\Delta C_{p}(T)$ has a much better agreement with the calorimetry data than do any of the $\Delta C_{V}(T)$ calculations shown in Fig. 2. The agreement between the measured and calculated $\Delta C_{p}(T)$ data is good, 
considering that there are no adjustable parameters in correlating the results from two very different sets of measurements-inelastic neutron scattering and differential calorimetry.

Thermal expansion was calculated from the phonon DOS curves by arranging Eq. (7) to solve for $\alpha$. At $300 \mathrm{~K}$, we obtained linear coefficients of thermal expansion of $15.5 \times 10^{-6} / \mathrm{K}$ for the partially disordered alloy, and $13.7 \times 10^{-6} / \mathrm{K}$ for the ordered alloy. These results seem reasonable, but we note that they would be more accurate if we used the larger bulk modulus obtained by electronic structure calculations $^{32,33}$ rather than the data of Table I.

\section{B. Microstructure and elastic energy}

Although the $\Delta C_{p}(T)$ calculated by our second attempt in Sec. V A with Eq. (13) was successful, the $\Delta C_{p}(T)$ calculated by our first attempt with Eq. (12) was qualitatively unsuccessful. We believe the problem in using Eq. (12) originates with our measured bulk moduli. We propose a microstructural mechanism for elastic energy storage in polycrystalline $\mathrm{Ni}_{3} \mathrm{~V}$ with the $D 0_{22}$ structure which allows for macroscopic differences in the bulk moduli of the two samples, even though they have nearly the same local chemical order. The mechanism is based on the tetragonal anisotropy of the thermal expansion of the ordered alloy. For $\mathrm{Ni}_{3} \mathrm{~V}$ with $D 0_{22}$ order, it is known that the microstructure within each prior fcc crystallite consists of lenticular packets comprising lamellas of ordered domains. The domains are mutually arranged in a microtwin-type microstructure to minimize the elastic energy. ${ }^{17,18,21,22}$ Such a microstructure can be optimized to minimize elastic energy at only one temperature, however. With changes in temperature, the ordered domains expand or contract anisotropically, generating internal elastic energy owing to geometrical mismatch between the lenticular packets. The ordered domains were originally formed during the annealing and cooling from $850^{\circ} \mathrm{C}$, so we expect the microstructure was optimized for minimizing elastic energy at high temperatures. Anisotropic thermal contraction upon cooling will generate internal stresses at room temperature. Tanner showed that the tetragonality of ordered $\mathrm{Ni}_{3} \mathrm{~V}$ can vary from about 1 to $2 \%$, depending on the thermal processing. ${ }^{17}$ (Our ordered alloy had a tetragonality of 1.7\%.) Nonequilibrium tetragonalities require the presence of internal stresses. In Tanner's work, significant strain contrast was found in transmission electron micrographs of ordered $\mathrm{Ni}_{3} \mathrm{~V}$ at room temperature, and others have reported large strain effects around $\mathrm{Ni}_{3} \mathrm{~V}$ precipitates in $\mathrm{Ni}-\mathrm{V}$ alloys. $^{21,22}$ In comparison, crystals of the disordered alloy have little or no tetragonality. Since the partially disordered alloys have a high degree of chemical order, however, they must have significant stored elastic energy at all temperatures. These local elastic distortions cause "tweed" diffraction contrast in TEM. We do not know the temperature dependence of these elastic distortions in the partially disordered alloy, although it is likely that they change with temperature.

We expect this microstructural mechanism for elastic energy storage in the polycrystalline ordered alloy to affect the elastic moduli measured in tests on macroscopic specimens. We expect the measured bulk modulus, $B \equiv V \partial^{2} E / \partial V^{2}$, to depend in the usual way on the energy of the crystal origi-

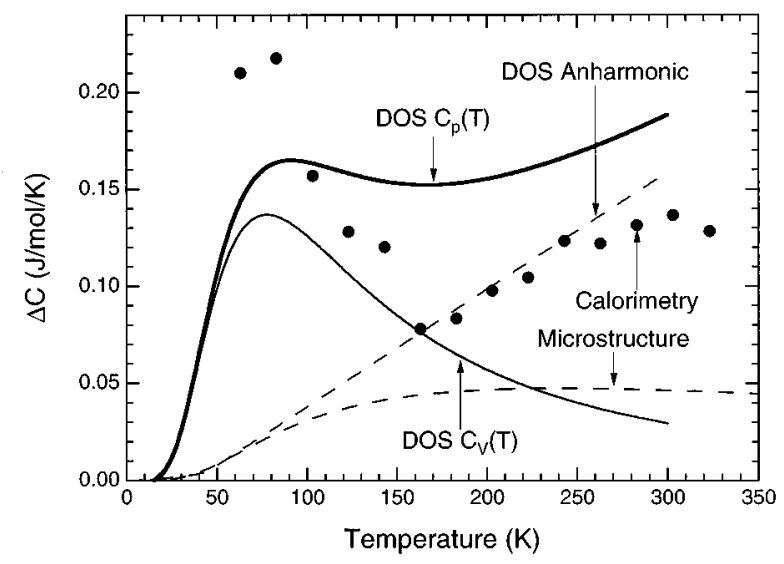

FIG. 6. Differential heat capacities. Curve DOS $C_{p}(T)$ and points calorimetry are as presented in Fig. 2. Curve DOS Anharmonic is calculated with the third term of Eq. (13) as described in the text. Curve Microstructure is calculated with Eq. (18) in the text, and is presented only as a functional form (with likely its opposite sign). Curve DOS $C_{p}(T)$ is the sum of the curves DOS $C_{V}(T)$ and DOS Anharmonic.

nating directly from the interatomic potential, $E_{x t l}$, but we expect an additional contribution from the elastic energy stored in the microstructure, $E_{\mu \mathrm{str}}$ :

$$
B=V\left(\frac{\partial^{2} E_{x t l}}{\partial V^{2}}+\frac{\partial^{2} E_{\mu s t r}}{\partial V^{2}}\right)=B^{\prime}+B^{\prime \prime}
$$

The second contribution, $B^{\prime \prime}$, originates with how the internal stresses are changed by the mechanical loading of the material. The sign of $B^{\prime \prime}$ is not obvious, because these elastic strains are added heterogeneously to the microstructure, and will interact with strains generated by thermal contraction. Furthermore, we do not expect a tetragonal crystal to be elastically isotropic. Our moduli seem low in comparison with those of electronic structure calculations. ${ }^{32,33}$ We therefore suspect that $B^{\prime \prime}$ is negative, and it becomes more negative for the partially disordered sample with its larger internal strains.

A microstructural contribution to the heat capacity is expected to originate with thermal contraction from the temperature $T_{H}$ where the microstructure was formed to the temperature $T$. The microstructural storage of elastic energy at a temperature $T$ will be

$$
E_{\mu \mathrm{str}}=\frac{B^{\prime \prime} \bullet}{2}\left(\int_{T}^{T_{H}} 3 \alpha\left(T^{\prime}\right) d T^{\prime}\right)^{2}
$$

The heat capacity associated with this microstructural energy, $\left.C_{p \mu s t r}(T)=\partial E_{\mu s t} / \partial T\right)_{p}$, can be calculated as a function of temperature. Using Eq. (10) to provide the temperature dependence for $\alpha(T)$ through the temperature dependence of $\Delta C_{V}(T)$, we obtain from Eq. (17)

$$
C_{p \mu \mathrm{str}}(T)=\frac{B^{\prime \prime} \gamma^{2}}{B^{\prime 2}} C_{V}(T)\left[E_{\mathrm{ph}}(T)-E_{\mathrm{ph}}\left(T_{H}\right)\right] .
$$

We do not know the prefactors of Eq. (18), except that we expect them to differ between the partially disordered and ordered materials. The functions $C_{V}(T)$ and $E_{\mathrm{ph}}(T)$ do not differ significantly between these two materials, so it is rea- 
sonable to calculate the temperature dependence of the difference in microstructural heat capacities: $\Delta C_{p \mu s \mathrm{str}}(T)$ $=C_{p \mu \mathrm{str}}^{\mathrm{PD}}(T)-C_{p \mu s \mathrm{st}}^{\mathrm{O}}(T)$. This temperature dependence is presented (probably with its opposite sign) in Fig. 6. It is perhaps not surprising that adding an appropriately scaled $\Delta C_{p \mu s t r}(T)$ to the $\Delta C_{p}(T)$ calculated from the phonon DOS curves in Sec. V A can improve the quality of fit to the experimental data. The improvement in the fit was not large enough to make a convincing case for the size of $\Delta C_{p \mu s t r}(T)$, however. By assuming the difference in $B^{\prime \prime}$ is the measured difference in Young's moduli (Table I), from Eq. (19) we obtain a difference in elastic energy of about 100 $\mathrm{J} / \mathrm{mol}$ at low temperatures, with the ordered alloy having the larger elastic energy. This estimate shows that $\Delta C_{p \mu \mathrm{str}}(T)$ could be comparable to the other contributions presented in Fig. 6. Unfortunately, although we expect that $\Delta C_{p \mu s t r}(T)$ is a real phenomenon, its quantitative evaluation is not justified given the accuracy of our calorimetry data and the uncertainty in the calculated anharmonic part of $\Delta C_{p \mu s t r}(T)$. Furthermore, the thermal contraction of the ordered material upon cooling from 1123 to $160 \mathrm{~K}$ is large, being about $1 \%$. With anisotropic contractions, it is likely that the elastic contractions may give way to some local internal plastic deformation at the lowest temperatures. Plastic deformation in the ordered alloy could alter $\Delta C_{p \mu \text { str }}(T)$ at the low temperatures.

\section{Microstructure and plastic deformation}

The ordered $\mathrm{Ni}_{3} \mathrm{~V}$ does not exhibit ideally elastic behavior, as shown by the mechanical hysteresis of Fig. 3. The area of the loop is largest for the first few cycles, but the loop remains relatively constant after the fifth cycle shown in Fig. 3 . The area of the loop increases strongly with the maximum strain. We are confident that the data of Fig. 3 show the hysteretic loss of mechanical energy in the ordered alloy is greater than in the partially disordered alloy (for which the maximum strain was larger, but the area of the loop is smaller). In a cyclic process, the mechanical deformation of the ordered alloy should be more strongly converted into heat. The area of the particular hysteresis loop shown for the ordered alloy in Fig. 3 corresponds to $0.6 \mathrm{~J} / \mathrm{mole}$, which seems too small to account for the large anharmonic effects in Fig. 2.

We are still investigating the origin of the large mechanical hysteresis in the ordered $\mathrm{Ni}_{3} \mathrm{~V}$. The hysteretic behavior of the ordered alloy could be promoted by the internal stresses from thermal contraction, which may be nearly large enough to drive plastic deformation at some locations in the microstructure. It is also possible that hysteretic effects may arise if the axes of tetragonality of the $\mathrm{D}_{22}$ structure change to accommodate the applied stress. We have performed preliminary measurements of x-ray diffraction patterns when the sample was under a compressive strain of about $0.2 \%$. We found no evidence of transformations between variants of the $\mathrm{DO}_{22}$ structure at this strain, however.

\section{Entropy}

Using the curve labeled DOS $C_{V}(T)$ in Fig. 6, we used Eq. (1) to calculate the difference in vibrational entropy of the partially disordered and ordered $\mathrm{Ni}_{3} \mathrm{~V}$ to be $0.025 \mathrm{k}_{\mathrm{B}} /$ atom at $300 \mathrm{~K}$, with the partially disordered mate- rial having the higher entropy. This calculated heat-capacity curve does lie below the experimental data at low temperatures, and above at higher temperatures. To make the estimate of the difference in vibrational entropy more consistent with the calorimetry data, we increased the curve DOS $\Delta C_{V}(T)$, and decreased the curve DOS Anharmonic, and with the sum of these new curves in Eq. (1) we obtained the same value of $\Delta S_{\text {vib }}(T)=(0.038 \pm 0.015) k_{B} /$ atom at $300 \mathrm{~K}$. The anharmonic contribution to the heat capacity provides a thermal increase of the difference in vibrational entropy of $5.84 \times 10^{-5} k_{B}$ atom ${ }^{-1} \mathrm{~K}^{-1}$. This difference in vibrational entropy between the partially disordered and ordered states of $\mathrm{Ni}_{3} \mathrm{~V}$ is smaller than results reported for other alloys. ${ }^{8-12} \mathrm{We}$ believe the reason is that our partially disordered $\mathrm{Ni}_{3} \mathrm{~V}$ had nearly the same chemical long-range order as did our ordered $\mathrm{Ni}_{3} \mathrm{~V}$.

\section{CONCLUSIONS}

We performed a comparative study of two states of $\mathrm{Ni}_{3} \mathrm{~V}$, a state of partial disorder obtained by rapid quenching, and the state of equilibrium $\mathrm{D}_{22}$ order obtained by annealing. The heat capacity of the partially disordered alloy was consistently larger than that of the ordered alloy in the temperature range of our calorimetry measurements, $60-325 \mathrm{~K}$. Inelastic neutron scattering was used to obtain approximate phonon density of states curves for the two alloys at 11 and $300 \mathrm{~K}$. The phonon energies were larger for the ordered alloy than the disordered alloy, as expected from their difference in heat capacities. The $C_{V}(T)$ calculated from the phonon DOS curves measured at $11 \mathrm{~K}$ could account for most of the difference in heat capacities of the two materials at temperatures below $160 \mathrm{~K}$.

The phonon DOS curves showed thermal softening between 11 and $300 \mathrm{~K}$. The phonon DOS of the partially disordered alloy showed more thermal softening than the ordered alloy, and this softening could account for much of the differential anharmonic heat capacity of the two states of $\mathrm{Ni}_{3} \mathrm{~V}$ at the higher temperatures.

We performed ancillary measurements of the linear coefficients of thermal expansion and the Young's moduli of the two alloys. The thermal expansion coefficients could be calculated from the temperature dependencies of the phonon DOS curves. There was a significant difference in the Young's moduli of the two alloys, however. With these moduli the classical anharmonic contribution, $C_{p}(T)-C_{V}(T)=9 B \backsim \alpha^{2} T$, predicted the wrong sign of the measured anharmonic contribution. This suggests that some of the anharmonic heat capacity could arise from a microstructural contribution. When the alloy is cooled from the high temperature where the ordered domains are formed, anisotropic thermal contractions cause the buildup of elastic energy in the ordered alloy. With increasing temperature, this elastic energy is relieved and converted into heat, suppressing the measured heat capacity. Our present data are unfortunately insufficient to isolate rigorously this microstructural contribution to the heat capacity. There may be another small microstructural contribution to the anharmonic heat capacity orginating with the hysteretic mechanical behavior of ordered $\mathrm{Ni}_{3} \mathrm{~V}$.

For the partially disordered and ordered alloys, we estimate the difference in vibrational entropy to be $\Delta S_{\text {vib }}(T)=(0.038 \pm 0.015) k_{B} /$ atom at $300 \mathrm{~K}$. The anharmonic contribution to the heat capacity provides a thermal increase of the difference in vibrational entropy with temperature of 
the amount $5.84 \times 10^{-5} k_{B}$ atom ${ }^{-1} \mathrm{~K}^{-1}$. The difference in the elastic energy in the microstructure is about $100 \mathrm{~J} / \mathrm{mol}$ at low temperatures, and may make a significant contribution to the difference in heat capacity of the partially disordered and ordered alloys.

\section{ACKNOWLEDGMENTS}

We thank M. Walter and G. Ravichandran for providing the ultrasonic facilities for the attempted sound velocity mea- surements, and R. D. Conner for help with the mechanical testing. L. Preister and I. Wong performed much of the sample preparation. We thank L. Anthony for valuable discussions. The Oak Ridge National Laboratory is managed for the Department of Energy by Lockheed Martin Energy Research, Oak Ridge, Tennessee under Contract No. DEAC05-96OR22464. This work was supported by the U.S. Department of Energy under Contract No. DE-FG-0396ER45572.
${ }^{1}$ J. C. Slater, Introduction to Chemical Physics (McGraw-Hill, New York, 1939), pp. 215-217.

${ }^{2}$ C. Booth and J. S. Rowlinson, Trans. Faraday Soc. 51, 463 (1955).

${ }^{3}$ P. J. Wojtowciz and J. G. Kirkwood, J. Chem. Phys. 33, 1299 (1960).

${ }^{4}$ H. Bakker, Philos. Mag. A 45, 213 (1982).

${ }^{5}$ J. A. D. Matthew, R. E. Jones, and V. M. Dwyer, J. Phys. F 13, 581 (1983).

${ }^{6}$ H. Bakker and C. Tuijn, J. Phys. C 19, 5585 (1986).

${ }^{7}$ J. K. Okamoto, C. Ahn, and B. Fultz, in Microbeam Analysis1990, edited by J. R. Michael and P. Ingram (San Francisco Press, San Francisco, 1990), pp. 56-58.

${ }^{8}$ L. Anthony, J. K. Okamoto, and B. Fultz, Phys. Rev. Lett. 70, 1128 (1993).

${ }^{9}$ L. Anthony, L. J. Nagel, J. K. Okamoto, and B. Fultz, Phys. Rev. Lett. 73, 3034 (1994).

${ }^{10}$ B. Fultz, L. Anthony, L. J. Nagel, R. M. Nicklow, and S. Spooner, Phys. Rev. B 52, 3315 (1995).

${ }^{11}$ B. Fultz, L. Anthony, J. L. Roberston, R. M. Nicklow, S. Spooner, and M. Mostoller, Phys. Rev. B 52, 3280 (1995).

${ }^{12}$ L. J. Nagel, L. Anthony, and B. Fultz, Philos. Mag. Lett. 72, 421 (1995).

${ }^{13}$ J. M. Sanchez, J. P. Stark, and V. L. Moruzzi, Phys. Rev. B 44, 5411 (1991).

${ }^{14}$ T. Mohri, S. Takizawa, and K. Terakura, J. Phys. Condens. Matter 5, 1473 (1993).

${ }^{15}$ S. J. Clark and G. J. Ackland, Phys. Rev. B 48, 10899 (1993).

${ }^{16}$ G. D. Garbulsky and G. Ceder, Phys. Rev. B 49, 6327 (1994).

${ }^{17}$ L. E. Tanner, Phys. Status Solidi 30, 685 (1968).

${ }^{18}$ A. François, G. Hug, and P. Veyssière, Philos. Mag. A 66, 269 (1992).
${ }^{19}$ P. A. Flinn, G. M. McManus, and J. A. Rayne, J. Phys. Chem. Solids. 15, 189 (1960).

${ }^{20}$ F. J. Rotella (unpublished); R. B. Von Dreele, J. D. Jorgensen, and C. G. Windsor, J. Appl. Crystallogr. 15, 581 (1982).

${ }^{21}$ H. A. Moreen, R. Taggart, and D. H. Polonis, Metall. Trans. A 5, 79 (1974).

${ }^{22}$ M. J. Pfeifer, P. W. Voorhees, and F. S. Biancaniello, Scr. Metall. Mater. 30, 743 (1994).

${ }^{23}$ J. Chevrier, J. B. Suck, M. Perroux, and J. J. Caponi, Phys. Rev. Lett. 61, 554 (1988).

${ }^{24}$ C.-K. Loong, P. Vashishta, R. K. Kalia, W. Jin, M. H. Degani, D. G. Hinks, D. L. Price, J. D. Jorgensen, B. Dabrowski, A. W. Mitchell, D. R. Richards, and Y. Zheng, Phys. Rev. B 45, 8052 (1992).

${ }^{25}$ J. Chevrier, J. B. Suck, J. C. Lasjanunias, M. Perroux, and J. J. Caponi, Phys. Rev. B 49, 961 (1994).

${ }^{26}$ V. F. Sears, Phys. Rev. A 7, 340 (1973).

${ }^{27}$ J.-B. Suck and H. Rudin, in Glassy Metals II, edited by H. Beck and H.-J. Güntherodt (Springer-Verlag, Berlin, 1983), Chap. 7.

${ }^{28}$ D. D. Klug, E. Whalley, E. C. Svensson, J. H. Root, and V. F. Sears, Phys. Rev. B 44, 841 (1991).

${ }^{29}$ H. R. Schober and P. H. Dederichs, in Numerical Data and Functional Relationships in Science and Technology, edited by K.-H. Hellwege, Landolt-Börnstein, New Series, Group III, Vol. III/ 13a (Springer-Verlag, Berlin, 1981).

${ }^{30}$ G. A. de Wit and B. N. Brockhouse, J. Appl. Phys. 39, 451 (1968).

${ }^{31}$ F. W. de Wette and A. Rahman, Phys. Rev. 176, 784 (1968).

${ }^{32}$ S. Pei, T. B. Massalski, W. M. Temmerman, P. A. Sterne, and G. M. Stocks, Phys. Rev. B 39, 5767 (1989).

${ }^{33}$ W. Lin, J-H. Xu, and A. J. Freeman, Phys. Rev. B 45, 10863 (1992). 